\title{
FRACTAL ANGLE BASED DIFFERENTIATION OF TERM PREGNANCIES USING UTERINE ELECTROMYOGRAPHIC SIGNALS
}

\author{
P. Vardhini and S. Ramakrishnan \\ Non-Invasive Imaging and Diagnostics Laboratory, Biomedical Engineering Group, \\ Department of Applied Mechanics, Indian Institute of Technology Madras, India, 600036
}

Corresponding Author: P. Vardhini

Email: niidvardhini@gmail.com

https://doi.org/10.34107/BiomedSciInstrum.57.04350

\begin{abstract}
Uterine Electromyography ( $\mathrm{uEMG}$ ) is a non-invasive technique that provides quantitative measure of uterine activity from the abdominal surface. In this work, an attempt has been made to investigate Term (gestational age $>37$ weeks) uEMG signals using Adaptive Fractal Analysis (AFA). For this, the signals obtained in second and third trimesters are considered and subjected to AFA. The fluctuation function is computed and the corresponding linear scaling regions are identified based on Chi-square statistic, standard error of slope, and coefficient of determination. Angle-based features from multiple scaling regions namely, inter-fractal angle and, short- and long-term fractal angles are extracted and are used for further analysis. The obtained results demonstrates that AFA approach can characterize the Term signals during varied gestational ages. All features show significant differences $(\mathrm{p}<0.05)$ in both groups. Feature values suggest that the third trimester signals possess more correlated and smoother fluctuations when compared to second trimester signals. This is attributed to the increased coordination of uterine contractions as delivery approaches. Hence, it appears that the proposed adaptive anglebased fractal features could be potential biomarkers in analyzing the muscle contractions associated with Term pregnancies.
\end{abstract}

Keywords: Uterine electromyography; Term pregnancy; Adaptive fractal analysis; Fractal angles

\section{INTRODUCTION}

Several physiological and emotional changes occur in women during pregnancy, in order to accommodate and nurture the developing fetus [1]. Monitoring uterine muscle contractions is crucial to analyze the adaptations of the women to physiological and anatomical changes and development of fetus. These contractions are due to the propagation of electrical activity in smooth muscle cells of the uterus, referred to as myocytes. Throughout the pregnancy, there are weak and inefficient contractions, as the uterus has a quiescent behavior. As it proceeds towards labor, the contractions are stronger and efficient for the delivery of fetus [2].

The current methods used in clinical practice for the evaluation of uterine contractions include manual palpation, Tocodynamometry (Toco) and Intrauterine Pressure (IUP) determination. However, due to the limitations such as need for constant presence of a trained clinician in manual palpation, low sensitivity of Toco method and invasiveness of IUP determination, these techniques are reported to be not favourable for the assessment of uterine activity [3]. Uterine Electromyography (uEMG) is a noninvasive procedure to record electrical activity of the uterus from surface of abdomen [1]. It provides an objective assessment of uterine contractions by examining the underlying electrical potentials. Using uEMG, frequency, duration and intensity of the uterine contractions could be evaluated. Hence, it is reported as a potential technique in pregnancy monitoring [3]. Further, uEMG signals can reveal changes in contractions preceding delivery. Several signal processing algorithms utilized for the analysis of uEMG are unable to address the complexity of such signals [4]. 